\title{
Understanding the Necessary Conditions of Multi-Source Trust Transfer in Artificial Intelligence
}

\author{
Maximilian Renner \\ Karlsruhe Institute of Technology \\ maximilian.renner@kit.edu
}

Scott Thiebes

Karlsruhe Institute of Technology

thiebes@kit.edu

\author{
Sebastian Lins \\ Karlsruhe Institute of Technology \\ lins@kit.edu
}

\author{
Matthias Söllner \\ University of Kassel \\ soellner@uni-kassel.de
}

\author{
Ali Sunyaev \\ Karlsruhe Institute of Technology \\ $\underline{\text { sunyaev@kit.edu }}$
}

\begin{abstract}
Trust transfer is a promising perspective on prevalent discussions about trust in AI-capable technologies. However, the convergence of AI with other technologies challenges existing theoretical assumptions. First, it remains unanswered whether both trust in AI and the base technology is necessary for trust transfer. Second, a nuanced view on trust sources is needed, considering the dual role of trust. To address these issues, we examine whether trust in providers and trust in technologies are necessary trust conditions. We conducted a survey with 432 participants in the context of autonomous vehicles and applied necessary condition analysis. Our results indicate that trust in AI technology and vehicle technology are necessary sources. In contrast, only vehicle providers represent a necessary source. We contribute to research by providing a novel perspective on trust in AI, applying a promising data analysis method to reveal necessary trust sources, and consider duality of trust in trust transfer.
\end{abstract}

\section{Introduction}

Trust has been a central concept in technology acceptance research for decades and has proven to be a key determinant of individuals' willingness to accept and use a technology because it mitigates uncertainties and risks related to vulnerabilities [1-3]. It is therefore not surprising that the question of how to establish trust in AI-capable technologies has become a core discussion in contemporary information systems (IS) research [e.g., 4, 5, 6]. By an AI-capable technology, we refer to a base technology that is augmented or automated with AI technologies (e.g., computer vision, natural language processing, or pattern recognition), and thus AI-capable technologies result from the convergence of AI with base technologies $[7,8]$. For example, AI more and more converges with vehicle technology (as base technology). This convergence results in AI-capable autonomous vehicles (AVs) that provide driver assistant and infotainment functions [9]. Several frameworks and guidelines to promote trust in AI have recently been developed and published by researchers, industry, and policymakers [e.g., 6, 10]. Likewise, research recently examined antecedents of trust in AI (e.g., explainable AI [11]) and analyzed the impact of trust on user beliefs and behaviors (e.g., user satisfaction with AI technologies [5]). While providing valuable contributions, extant research has neglected to consider trust transfer processes to establish trust in AI-capable technologies. Trust transfer theory proposes that users' trusting beliefs in already existing and familiar sources (e.g., a technology or a person) may transfer to a novel and unknown target $[12,13]$. Such trust transfer typically results if users perceive a strong relationship between a familiar source and an unknown target $[12,13]$. We argue that trust transfer processes are also likely to occur in the case of novel AI-capable technologies because they result from the convergence of AI with one or more base technologies that, as trust sources, are typically known by users [14]. For example, if AI technology is embedded in the vehicle technology, leading to AI-capable AVs, users may transfer their established trust in familiar vehicle technologies and supposedly also transfer trust in related AI technologies (e.g., virtual assistants like Alexa or Siri) to unknown AVs.

However, the convergence of AI and base technologies challenges existing theoretical assumptions of trust transfer for two reasons. First, trusting AI-capable technologies may necessitate multiple sources of trust transfer, meaning that users may require both trusting beliefs in a base technology (e.g., vehicle technology) and in AI to trust the unknown AI-capable technology. Although recent research has already validated multisource trust transfer in related contexts [e.g., 15], AIspecifics put in doubt whether a trust transfer from $\mathrm{AI}$ is 
achievable. In particular, trust transfer requires users to be familiar with AI as a trust source $[12,13]$. Yet, users may still lack experience or profound knowledge of extant AI technologies due to their novelty and complexity, among others [16]. It remains of high interest to understand if users' trusting beliefs in both trust sources are necessary (i.e., in the base technology and AI) or whether trust in one source is sufficient to achieve trust transfer in an unknown AI-capable technology.

Second, extant research on trust transfer has mostly focused on either a known technology or provider as a source for trust transfer [e.g., 12, 17]. On the contrary, trust research proposes that users' trust typically takes an interwoven dual role: trust in a provider and trust in a technology that must be considered in parallel $[18,19]$. The duality of trust is particularly relevant in the context of AI because users may lack an understanding of concrete AI but may be familiar with the AI providers behind them, such as Google, Microsoft, IBM, or Amazon. For example, Alphabet (i.e., Google) now cooperates with Daimler concerning AI-capable autonomous trucks. Users of AVs may be familiar with Alphabet but not with the specific AI technology used to augment vehicle technology. From a trust transfer perspective, it remains unanswered whether both trust in providers and trust in technologies as trust sources are necessary to establish trust in an AI-capable technology. A more nuanced perspective on multi-source trust transfer is therefore warranted in the context of AI-capable technologies, which considers trust transfer of both providers and technologies to enable the comparison of necessary trust conditions. We therefore seek to answer the research question (RQ):

$R Q:$ What are the necessary trust sources in multisource trust transfer into AI-capable technologies from a dual trust perspective (i.e., trust in technologies and trust in providers)?

To answer our RQ, we ground our research in trust transfer theory $[12,13]$ and develop a theoretical model focusing on multi-source trust transfer while taking a dual trust perspective and discussing whether both trust sources are needed [19]. Specifically, we focus on the context of trust transfer from known vehicle technologies and AI to unknown AVs. We tested our theoretical model by conducting an online survey among 432 participants and performing a necessary condition analysis (NCA) [20].

Our results confirm that trust in vehicle technologies and trust in AI technologies form necessary sources to transfer trust in AVs. On the provider level, however, our study leads to counterintuitive findings: only trust in vehicle providers is perceived as a necessary source, whereas trust in AI providers does not meet the necessary condition requirement. This study contributes to the literature in three key areas. First, we provide a novel theoretical perspective on establishing trust in converging AI-capable technologies by suggesting the presence of trust transfer processes in the context of AI. Second, we contribute to trust transfer theory by showing the importance of the duality of trust, indicating that trust transfer processes emerge from both a provider and technology trust perspective. Third, we highlight the usefulness of conducting NCAs, introduced by Dul [20], to understand which trust sources are a necessary condition in multi-source trust transfer.

\section{Background}

\subsection{Trust in autonomous vehicles}

A common research case of converging AI with base technologies is AI-enhanced driving functionalities in AVs [4]. The step-by-step convergence of AI with vehicle technology is typically divided into six levels of automation [21]. Level zero defines a vehicle without automated functionalities and thus without AI capabilities. While the level of automation increases, the amount and variety of AI capabilities also increase to support autonomous driving functionalities [9]. For example, in intermediate levels of automation, AI supports the driver with a range of functionalities, such as lane-keeping assistance, speed control, or infotainment systems, whereas the drivers continue to be responsible and in control of their vehicles. With higher levels of automation, AI takes over more and more actions for the drivers, allowing them to relinquish control of their vehicle to the AI in predefined situations (e.g., on specially upgraded highways). At level five, convergence of AI and the vehicle is most advanced, and AI enhances intelligent automation to match the capabilities of human drivers in most driving scenarios.

However, the convergence of AI and vehicle technologies is a double-edged sword and not only brings advantages (e.g., optimizing traffic or cost-efficient share mobility [9]) but also drawbacks: the use of AVs contains high physical risks such as accidents at high speeds; and it reflects a step-change from augmentation, where users collaborate closely with AI-capable technology, to automation, where technology is completely taking over complex human tasks. Consequently, it is important to understand how people establish trust in AVs and what trust conditions are necessary for the trust-building process [4].

\subsection{Duality of trust}

Nowadays, most IS research adopts a dual perspective on trust. First, trust in people or organizations [18, $22]$, such as trust in a provider [2], team members [23], 
or vehicle providers. Second, trust in technology or, more specifically, in an IT artifact [18, 22], like a cloud service [19] or vehicle technology. Trust in people and trust in technology not only differ on the underlying object but also regarding the trusting beliefs. Interpersonal trusting beliefs reflect judgments that the other party has appropriate attributes and motives to behave as expected in a risky situation [24], whereas technology-related trust reflects beliefs about a technology's characteristics rather than of its motives [18]. Previous research agrees that individuals can change their expectations about a person's competence (i.e., their ability to do what the individual needs), benevolence (i.e., their care and motivation to act in the individual's interests), and integrity (i.e., their honesty and promise-keeping) [25]. By contrast, trust in a technology typically refers to the functionality of the technology (i.e., providing features needed to complete a task), its helpfulness (i.e., help functionalities will provide necessary advice), and its reliability (i.e., technology will consistently operate properly) $[18,26]$. In the case of AI-capable technologies, both lenses on trust may play a decisive role because users may lack knowledge about AI technologies but may be familiar with their providers or vice versa [6]. Although trust may be established based on users' perceptions toward its technological functionalities and its provider, the question is whether both trust perspectives are necessary to transfer users' trust into an unknown AI-capable technology. We therefore look at trust transfer theory.

\subsection{Related research on trust transfer}

In its essence, trust transfer theory explains the relationship between an already known trusted source and a novel, unknown target $[12,13]$. Extant research indicates that users' trust in a trusted and familiar source can be transferred to a relatively unknown target under the condition that the target has a strong relationship with the trusted source [12]. Thus, trust transfer can be characterized as a fundamental form of trust adjustment between two objects. For example, if users perceive the relationship between a source and a target as close and strong, the transferability of trust is more likely to happen. In contrast, users may not trust the target if the source-target relationship is perceived as weak.

In general, research on trust transfer remains scarce (refer to Gong et al. [17] for a recent review) and has not been applied to the context of AI-capable technologies. Exploring whether trust transfer also applies to AI contexts provides a promising perspective on prevalent discussions about trusting AI and supports researchers in better theorizing the emergence of trust in AI-capable technologies. Nevertheless, understanding trust transfer into converged technologies remains challenging given its multi-source character and the duality of trust.

Regarding the multi-source character, prior research has shown that trust transfer can appear in both a single-source context (e.g., from trust in web payment services to trust in mobile payment services [17]) and a multi-source context (e.g., from trust in public administration and the Internet to the public e-service [27]). In particular, prior research has analyzed whether an increase in trusting beliefs toward a source lead to higher trust in a target. The interpretation of relationships between the source and the target, therefore, follows a sufficiency logic, meaning that a determinant (e.g., trust in AI) may be sufficient to produce the outcome (e.g., trust in AV) [20, 28]. However, it may not be necessary, and thus the absence of one trust source could be compensated by another trust source. For example, trust in vehicle technologies may compensate a lack of trust in AI technologies.

In contrast to sufficiency logic, necessity logic implies "that an outcome-or a certain level of an outcomecan only be achieved if the necessary cause is in place or is at a certain level" [28]. Coming back to our example, both trust sources may be necessary to achieve trust in AV. Taking such a necessity logic perspective is promising because it helps to identify must-have factors that must be satisfied to achieve a certain outcome [28]. In the context of trust transfer, taking a necessity logic thus clarifies which trust sources are needed to achieve trust in an unknown target, which has, however, been neglected so far in extant trust transfer literature.

Considering the highlighted importance of the duality of trust in an AI-capable technology context, a more nuanced view on necessary trust sources is needed. Reviewing trust transfer literature reveals extant research has either focused on technology trust transfer (e.g., trust in websites, e-WOM services, and web shopping services [17]), or interpersonal trust transfer (e.g., trusted members and the community [29]) but to the best of our knowledge not on both at the same time. We thus lack a clear understanding of whether trust transfer is possible simultaneously at the technology and provider level and which of these trust sources are necessary. As a result, further research is required that considers the trust duality in multi-source trust transfer.

\section{Research model}

To understand which trust sources are a necessary condition for trust in an unknown target, we first reflect on trust transfer mechanisms. Trust transfer's basic premise is a unique categorization process in that users' trusting beliefs toward a source could be extended to their trusting beliefs toward a target through categorybased processing $[12,13]$. Users typically place objects 
in different categories to classify, interpret, and understand their information about these and related objects [30]. A category is a set of systems, persons, products, or other entities that appear, to the user, related in some way. For example, users may assign Google, Amazon, and Microsoft to the category 'AI provider'. By grouping objects together that are alike in important respects, users enhance information processing efficiency and cognitive stability [31]. A key construct in theoretical accounts of categorization and trust transfer is similarity because it moderates the transfer of cognitive beliefs from one stimulus to another [30]. In order for trust to be transferred from a source to the target, users have to identify a close similarity between the source and the target object. If a source is similar to the target object, users are likely to assign the target object in the same category as the source, and transfer knowledge, affect, and intentions to the lesser-known target object $[12,30]$. Prior research on trust transfer and categorization has conceptualized similarity as having a strong business relationship or offering similar technology functionality, among others [17]. These conceptualizations align with the duality of trust, proposing that an interpersonal trust transfer emerges in case of a strong business relationship and technology trust transfer in case of similar functionality, respectively.

First, taking an interpersonal trust perspective, users will put the vehicle provider, AI provider, and AV provider in the same category if users perceive their association and a strong business tie [32]. For example, if users trust organization $\mathrm{A}$ and perceive that organizations $\mathrm{A}$ and $\mathrm{B}$ are partners, users will trust organization $\mathrm{B}$ as well to experience cognitive balance [32]. Although vehicle providers may build the AV themselves, including the intelligent autonomous driving functionalities, more and more providers are taking a different approach in practice and start collaborative projects with experienced and familiar AI providers. Thus, most AV providers are a joint partnership between an existing vehicle provider and AI provider. One example is the association of Mercedes with Waymo, which is owned by Alphabet. To this end, AI, and vehicle providers commonly converge to form AV providers. In line with trust transfer theory, we presume that trust transfer only emerges if users put the AV provider and the AI and vehicle provider in the same category, for example, when users recognize their mutual cooperation to offer the AVs. Since users may be already familiar with AI and vehicle providers, users may then swiftly become familiar with the AV provider, especially when they perceive a strong relationship. Given the interwoven interplay of the AI and vehicle providers to offer AVs, we argue that the users must have faith in both the AI and vehicle providers' competence, integrity, and benevolence to trust the (converged) AV provider. In contrast, if users believe that, for instance, an AI provider sells personal driving information to a third party (i.e., having low integrity), such as GPS locations of the vehicle, the categorization processes are hampered. Consequently, the user will not transfer trust to the AV provider, which is why we believe that trust in both providers is a necessary condition to establish trust in the target provider. Thus, we hypothesize:

Hla: Users' trust in vehicle providers is necessary for users' trust in AV providers.

H1b: Users' trust in AI providers is necessary for users' trust in AV providers.

Second, taking a technology trust perspective, trust transfer research argues similarly that users' trust is transferred based on the perceived technology similarity $[12,13]$. Users may put the source technology and the target technology into one category based on similar technology functionality. In the context of AVs, we argue that users will put vehicle technology and AV technology into the same category because it provides similar mobility functionalities. AVs will continue to consist of wheels, breaks, and a similar driving equipment, while initially retaining the steering wheel and pedals for the possibility of driver interactions. With higher levels of automation, more and more AI functionalities will be added, such as voice assistants or the possibility of autonomous driving based on intelligent automation without driver interactions [4]. These technological functionalities are similar to related AI-capable technologies, such as voice assistants in the home environment [e.g., 33] or intelligent automated customer chatbots [e.g., 34]. If users perceive technology similarities between AVs and their sources (i.e., vehicle technologies and AI technologies), they may put them into the same category, such as 'trustworthy technologies'. In contrast, if users have, for instance, high trust in vehicle technologies but doubt about an AI technology's reliability to provide safe automated-driving functions. Then, users may have less faith in AV's performance, contrasting their trust in vehicle technologies, ultimately disturbing the categorization and trust transfer process. Thus, we propose that trust in AI technologies and vehicle technologies are both necessary conditions to transfer trust in AV technologies and hypothesize:

H2a: Users' trust in vehicle technologies is necessary for users' trust in AV technologies.

$H 2 b$ : Users' trust in AI technologies is necessary for users' trust in AV technologies. 


\section{Research approach}

\subsection{Overview of necessary condition analysis}

We perform necessary condition analysis (NCA) to test our hypotheses - a research method that was recently developed and is increasingly applied in the IS discipline. Given its novelty, we first provide a brief overview of the method.

NCA was originally introduced by Dul in 2016 [20] to enable the identification of necessary conditions in data sets [20]. The uniqueness of NCA is that it reveals areas in scatter plots of dependent and independent variables that may indicate the presence of a necessary condition instead of analyzing the average relationships [28]. Unlike ordinary least squares-based regression techniques that produce a dashed line through the center of the relevant data points, such as PLS-SEM, NCA determines a ceiling line on top of the data [28]. The ceiling line separates the space with observations from the space without observations, whereas two default ceiling lines are available: (1) the ceiling envelopment-free disposal hull (CE-FDH) line, which is a nondecreasing step-wise linear line (step function); and (2) the ceiling regression-free disposal hull (CR-FDH) line, which is a simple linear regression line through the CE-FDH line [20].

For a variable to be a necessary condition, the empty space is decisive, whereas the larger the empty space, the larger the constraint of a variable on another. Each variable can be assessed in detail using a bottleneck table (e.g., Table 4 in Section 4.2.5). For the analysis with NCA, two key parameters are important: ceiling accuracy (c-accuracy) and necessity effect size d. The c-accuracy provides the number of observations that are on or below the ceiling line divided by the total number of observations and multiplied by 100 . While the c-accuracy of the CE-FDH is $100 \%$ per definition, it may be below $100 \%$ for the CR-FDH. Although there is no specific rule for an acceptable level of c-accuracy, estimating with a benchmark value (e.g., 95\%) is recommended [20]. The necessity effect size $d$ is calculated by dividing the ceiling zone (i.e., empty space) by the scope (i.e., space containing observations). While $\mathrm{d}$ ranging between $0 \leq \mathrm{d} \leq 1$, a small effect is characterized as $0<\mathrm{d}<.1$, a medium effect as $.1 \leq \mathrm{d}<.3$, a large effect as $.3 \leq \mathrm{d}<.5$, and a very large effect as $\mathrm{d} \geq .5$. Previous studies agreed on an effect size threshold of at least $d \geq$ .1 (at least a medium effect) to accept necessary conditions hypotheses [e.g., 28, 35]. Finally, to evaluate the significance of the meaningfulness of the effect size, a permutation test has to be considered when analyzing a necessary condition [36].
However, NCA is limited to only analyzing relationships between observable characteristics (e.g., regarding scales and the absence or presence of characteristics) or researchers' created indices (e.g., an index of business performance) [28]. With the help of computing factor scores or composite scores (e.g., via PLS-SEM), the NCA can be extended to measure unobservable, latent concepts, such as user satisfaction, use intention, and perceived usefulness [28]. To address this condition, it is therefore recommended to use the composite scores of PLS-SEM [28], while their generation considering the context of the structural model [37]. Using the indicator weights as input, PLS-SEM computes composite scores for each construct as linear combinations of the corresponding indicators, which have shown good reliability [38].

\subsection{Necessary condition analysis application}

We followed the steps proposed by Ringle et al. [28] to perform the NCA. First, we developed a survey to test our hypotheses (Sections 4.2.1-4.2.2). Second, we conducted the cross-sectional survey using online panel data provided by Amazon Mechanical Turk (Section 4.2.3). Using online panel data has been shown to be suitable for studying trust-related phenomena [e.g., 34, 39]. Research has demonstrated that the results of surveys using MTurk have high reliability and provide high-quality data comparable to student samples or online convenience samples [e.g., 40]. We restricted potential participants to those with a high reputation (at least $95 \%$ approval ratings and at least 5,000 conducted tasks) to ensure sufficiently high data quality [41]. We restricted participation to US workers to reduce cultural biases, and ensured minimum fair payment of participants (i.e., federal minimum wage of $\$ 7.25$ per hour). Afterward, we prepared and checked the data, evaluated the reliability and validity of the measurement models (Section 4.2.4). Next, we generated the latent variables scores using SmartPLS software, version 3.3.3 [42] and transferred them to $\mathrm{R}$ to perform the NCA (Section 4.2.5). Due to the fact that the c-accuracy of all variables is above $95 \%$, we used the CR-FDH line due to its better handling of outliers and measurement errors [20].

4.2.1. Survey procedures. We used six steps to collect survey data. First, we provided a short description of the study's objective, context, and examples of AI technologies (i.e., virtual assistants, recommender systems). Second, we asked subjects to think of a trustworthy AI provider and its provided AI technology they know and like, since familiarity with the source technology is required to enable trust transfer $[12,13]$. We asked subjects to name the AI provider they thought of or select 
one in a list provided by us (i.e., Microsoft, Apple, Amazon, Google, IBM Watson). We then measured subjects' trust perceptions toward the AI provider and its AI technology. Analog to this, subjects should next think of a trustworthy car manufacturer and its cars they know and like, and name it or select one of the provided ones (i.e., Toyota, Ford, VW, Tesla). Note that we narrowed down the area of vehicles to "cars" within the survey to achieve higher subject comprehensibility. Afterward, we measured subjects' trust in the car manufacturer and its car technology. Third, we added an attention check to control for continued attention and created a washout period between the measurement of our independent and dependent variables by letting subjects read an unrelated text and click on a hidden link [43]. Fourth, we introduced subjects into a scenario where they should consider the fictional example that their employer provides them with a company car as part of their salary. They had two options, whereas they could choose from a car with conventional technology and a car with AI-enabled autonomous driving technology. We provided the subjects with brief information about the autonomous car provider (i.e., a cooperation that has formed between the AI provider and car manufacturer to illustrate business tie-strength) and the technology (i.e., autonomous car technology takes over the complete control of the autonomous car when driving on the highway and providing further driver assistant functionalities to illustrate technical consistency). Fifth, we measured our dependent

Table 1. Measurement Items

\begin{tabular}{|l|l|c|}
\hline Label & Item & \multicolumn{1}{c|}{ Loading } \\
\hline Trust in Provider [Vehicle / AI / AV] [23] \\
\hline TP1 & $\begin{array}{l}\text { Overall, I feel that I can trust [Ve- } \\
\text { hicle Manufacturer / AI Provider / } \\
\text { AV Provider] completely. }\end{array}$ & {$[.818 / .884 / .922]$} \\
TP2 & $\begin{array}{l}\text { I feel comfortable depending on } \\
\text { [Vehicle Manufacturer / AI Pro- } \\
\text { vider / AV Provider] for the com- } \\
\text { pletion of AI-supported tasks. }\end{array}$ & {$[.879 / .907 / .931]$} \\
\hline & $\begin{array}{l}\text { I am comfortable letting [Vehicle } \\
\text { Manufacturer / AI Provider / AV } \\
\text { Provider] take responsibility for } \\
\text { tasks which are critical to [Vehicle } \\
\text { / AI / AV Technology] even when } \\
\text { TP3 cannot control them. }\end{array}$ & {$[.864 / .886 / .908]$} \\
\hline Trust in Technology [Vehicle / AI / AV] [18]
\end{tabular}

variables, namely trust in the AV technology and provider. Finally, we collected control variables and demographics.

4.2.2. Survey measures. We followed methodological recommendations and used previously validated scales for measuring the constructs in our survey (refer to Table 1). To measure individuals' trust in technology, we adopted measures from McKnight et al. [18] and individuals' trust in providers from Staples and Webster [23]. Note that we adapted and rephrased measurement items to fit our context and inserted the name of the proposed or selected vehicle manufacturer and AI provider (i.e., "I feel comfortable depending on VW for the completion of driving"). We also added items to measure a latent marker variable (i.e., "Music is important to my life"; "Bears are amazing animals"; "I find rugby interesting"; "When it comes to art, I prefer paintings over photography") [44].

4.2.3. Descriptive statistics. We recruited 432 participants, of which we removed 53 responses because 31 participants failed attention checks and 22 participants rushed through the survey. This process resulted in 379 valid responses. This number exceeds the approximate sample size of 198, which we calculated using the tool $\mathrm{G}^{*}$ Power (power $=.95$, effect size $\mathrm{f}^{2}=.1$ ) [45] as well as the median sample size of 200 from prior SEM studies [46]. More men (32.5\% females) participated in our survey, and participants were, on average, 30.4 years of age (minimum 23 years, maximum 67 years). Most participants had a high school $(18.5 \%)$ or undergraduate degree $(62.8 \%, 13.2 \%$ graduate degree), have held a driver's license for more than 5 years $(86.3 \%$, no driver license $1.3 \%$ ), had a vehicle which is 3 to 5 years $(26.6 \%)$ or over 5 years $(47.2 \%)$ old and used their vehicle daily $(64.4 \%)$ or weekly $(29.6 \%)$. On average, participants indicated that they often interacted with AI technologies (60.7 on a 100-point sliding scale) and rated the realism of the scenario with 82.5 on a 100 point sliding scale.

4.2.4. Data analysis and results. First, we assessed the measurement model. We assessed univariate and multivariate normality of the measurement items in our survey. One trust in vehicle technology item had the highest absolute skewness value of 2.091 (i.e., TT4), falling below the acceptable threshold of 3.0 for skewness [46]. Regarding the highest absolute kurtosis value, items of trust in technology for vehicle (i.e., TT4), AI, and AV (i.e., TT7) exceed the threshold 10.0 for kurtosis [46], 
Table 2. Measurement assessment

\begin{tabular}{|l|c|c|c|c|c|c|c|c|}
\hline \multirow{2}{*}{ Construct } & \multirow{2}{*}{$C R$} & \multirow{2}{*}{ AVE } & \multicolumn{6}{|c|}{ Fornell-Larcker Criterion } \\
\cline { 4 - 9 } & & & 1 & 2 & 3 & 4 & 5 & 6 \\
\hline $\begin{array}{l}1 . \text { Trust in } \\
\text { AV technology }\end{array}$ & .947 & .780 & .883 & & & & & \\
\hline $\begin{array}{l}\text { 2. Trust in } \\
\text { AV provider }\end{array}$ & .944 & .848 & $\begin{array}{c}.813 \\
(.882)\end{array}$ & .921 & & & & \\
\hline $\begin{array}{l}\text { 3. Trust in } \\
\text { vehicle provider }\end{array}$ & .890 & .729 & $\begin{array}{c}.514 \\
(.592)\end{array}$ & $\begin{array}{c}.508 \\
(.588)\end{array}$ & .854 & & & \\
\hline $\begin{array}{l}\text { 4. Trust in } \\
\text { vehicle technology }\end{array}$ & .944 & .739 & $\begin{array}{c}.496 \\
(.532)\end{array}$ & $\begin{array}{c}.320 \\
(.340)\end{array}$ & $\begin{array}{c}.738 \\
(.845)\end{array}$ & .860 & & \\
\hline $\begin{array}{l}\text { 5. Trust in } \\
\text { AI provider }\end{array}$ & .921 & .796 & $\begin{array}{c}.471 \\
(.522)\end{array}$ & $\begin{array}{c}.530 \\
(.592)\end{array}$ & $\begin{array}{c}.603 \\
(.713)\end{array}$ & $\begin{array}{c}.455 \\
(.499)\end{array}$ & .892 & \\
\hline $\begin{array}{l}\text { 6. Trust in } \\
\text { AI technology }\end{array}$ & .944 & .738 & $\begin{array}{c}.581 \\
(.622)\end{array}$ & $\begin{array}{c}.447 \\
(.478)\end{array}$ & $\begin{array}{c}.638 \\
(.713)\end{array}$ & $\begin{array}{c}.653 \\
(.697)\end{array}$ & $\begin{array}{c}.669 \\
(.738)\end{array}$ & .859 \\
\hline
\end{tabular}

which we then removed to ensure that the distributions of our measurement items do not deviate significantly from normality. We also controlled for data outliers and removed two observations exposing extreme outlines (z-score $>3$ ) in trust in vehicle technology.

Second, we assessed the constructs' reliability, convergent validity, and discriminant validity (refer to Table 2). All indicators fulfilled the minimum loading requirements (significance and load value) between the indicator and its latent construct, achieving convergent validity. The average variance extracted (AVE) was higher than the suggested minimum of .50 [47]. The composite reliability (CR) values were above .70 , demonstrating good internal consistency [48]. Regarding discriminant validity, the square root of each construct's AVE exceeded the inter-construct correlations. In addition, we measured the heterotrait-monotrait (HTMT) ratios of correlations. The HTMT between trust in AV technology and AV provider (.88) slightly exceeds the recommended threshold of .85 [38]. We decided to keep both constructs in our model because the Fornell-Larcker Criterion and the less conservative HTMT threshold of .90 are met, and more importantly, because prior theory has already acknowledged a strong relationship between trusting beliefs in technology and provider [e.g., 25]. We also examined variance inflation factor (VIF) values to test for multicollinearity in our data. All VIF values were below the threshold of 5, except for TT3 in case of trust in AV technology (i.e., VIF = 6.185), which we then removed to ensure that our data is not subject to severe multicollinearity issue [49].

Third, we account for common method variance (CMV) not only ex-ante through the careful design of the questionnaire, applying the recommendations of Podsakoffet al. [50]), but also ex-post by running a measured latent marker variable (MLMV) test and performing a construct level correction [44] relying on PLS-SEM and SmartPLS software, version 3.3.3 [42]. We added a CMV construct comprising the four MLMV items for each construct, modeled them as impacting each model construct, and compared the bootstrapping results. The differences in the path coefficients between the model constructs were found to be very small $(<.200)$ [51], and we, therefore, conclude that a potential CMV does not pose a significant threat to our results.

Table 3. NCA effect sizes

\begin{tabular}{|l|c|c|c|}
\hline & \multicolumn{3}{|c|}{ Trust in AV provider } \\
\hline Construct & $C R-F D H(d)$ & $p$-Value & $c$-accuracy \\
\hline Trust in AI technology & .121 & $<.001$ & $98.7 \%$ \\
\hline Trust in vehicle technology & .255 & $<.001$ & $98.1 \%$ \\
\hline & \multicolumn{3}{|c|}{ Trust in AV technology } \\
\hline Construct & $C R-F D H(d)$ & $p$-Value & c-accuracy \\
\hline Trust in AI provider & .030 & .264 & $99.7 \%$ \\
\hline $\begin{array}{l}\text { Trust in } \\
\text { vehicle provider }\end{array}$ & .286 & $<.001$ & $98.7 \%$ \\
\hline
\end{tabular}

4.2.5. NCA data analysis and results. The NCA's results (see Table 3 ) show a sufficiently high c-accuracy (c-accuracy $>95 \%$ ) and indicate that for trust in AV technology, both trust sources are meaningful and significant necessary condition $(\mathrm{d}>.100, \mathrm{p}<.001)$. Thus, trust in AI technology and trust in vehicle technology have a medium effect on trust in AV technology, supporting H2a and H2b. For trust in AV provider, however, only trust in vehicle provider is meaningful and a significant necessary condition $(\mathrm{d}=.286$, medium effect, $\mathrm{p}<.001$ ), supporting H1a. In contrast, trust in AI provider is not significant $(\mathrm{d}=.030, \mathrm{p}=.264)$, not supporting H1b.

\section{Table 4. Bottleneck table (percentages)}

\begin{tabular}{|l|c|c|}
\hline & Trust in AI technology & Trust in vehicle technology \\
\hline \multicolumn{3}{|c|}{ Bottleneck Trust in AV technology } \\
\hline $0 . .20$ & $\mathrm{NN}$ & $\mathrm{NN}$ \\
\hline 30 & $\mathrm{NN}$ & 0.6 \\
\hline 40 & $\mathrm{NN}$ & 10.8 \\
\hline 50 & 1.9 & 21.0 \\
\hline 60 & 10.8 & 31.2 \\
\hline 70 & 19.7 & 41.5 \\
\hline 80 & 28.6 & 51.7 \\
\hline 90 & 37.5 & 61.9 \\
\hline 100 & 46.3 & 72.1 \\
\hline \multicolumn{3}{|c|}{ Trust in AI provider } \\
\hline Bottleneck Trust in AV provider \\
\hline $0 . .20$ & $\mathrm{NN}$ & Trust in vehicle provider \\
\hline 30 & $\mathrm{NN}$ & $\mathrm{NN}$ \\
\hline 40 & $\mathrm{NN}$ & 7.3 \\
\hline 50 & $\mathrm{NN}$ & 16.8 \\
\hline 60 & $\mathrm{NN}$ & 26.3 \\
\hline 70 & $\mathrm{NN}$ & 35.8 \\
\hline 80 & $\mathrm{NN}$ & 45.3 \\
\hline 90 & 14.0 & 54.8 \\
\hline 100 & 36.7 & 64.2 \\
\hline
\end{tabular}

Each necessary condition can be assessed in detail with the bottleneck tables [28]. The bottleneck table represent an alternative form of the ceiling line results while it specifies the level of trust in a source that is necessary for a certain level of trust in a target. For example, 
Table 4 highlights that in order to reach a $60 \%$ level of trust in AV technology, two necessary conditions need to be in place: trust in AI technology at no less than $10.8 \%$ and trust in vehicle technology at no less than $31.2 \%$. In contrast, to reach a $60 \%$ level of trust in AV provider, only one necessary condition needs to be in place: trust in vehicle provider at no less than $35.8 \%$.

\section{Discussion}

\subsection{Principal findings}

In this study, we investigated what trust sources are necessary for trust transfer in a multi-source context. Following trust transfer theory, we were able to show that users perceive a strong similarity between vehicle and AV technology, leading to trust transfer $[12,13]$. Similar to current understandings that AVs still resemble vehicles based on recognizable mobility functionalities and interior/exterior design [e.g., 4, 9], we show that trust in vehicle technologies is a necessary condition for transferring trust in AV technologies. Our results indicate that trust in vehicle providers is also a necessary condition because users continue to perceive vehicle providers and AV providers as similar and thus put them into the same category.

Surprisingly and against our propositions, users seem to be hesitant to associate AI with AV technology. Although we could identify a medium effect of trust in AI technology on trust in AV technology, the medium effect of trust in vehicle technology on trust in AV technology was stronger than the effect of trust in AI technology on trust in AV technology. This may be the case because autonomous driving functionalities do not yet have a high market penetration [4] and users may still be skeptical due to trust-related issues of these AI-enabled functionalities [9]. Thus, users seem to perceive the link between trust in AI technology as a week but necessary source condition for trust in AVs. Counterintuitively, we could not confirm our hypothesis regarding the necessity of trust in AI providers for trust in AV providers. Eventually, users do not yet seem to associate AI providers with vehicle development. Similar to the technology perspective, the reason for this may be that the focus is still on the vehicle and not on the autonomous driving functionalities, and thus, the importance and necessity of the traditional vehicle providers remains. Although the new autonomous driving functionalities are emerging due to the convergence of AI with vehicles [6, 14 ], users still seem to trust a vehicle provider more in developing these driving-related functionalities. However, we show that despite the lack of trust in one source (i.e., trust in AI providers), it is still possible to transfer trust to the target, given at least trust in the other source (i.e., trust in vehicles).

\subsection{Theoretical and practical contributions}

From a research perspective, our study yields several important contributions. First, we provide a novel theoretical lens on establishing trust in new converging AI-capable technologies by suggesting the presence of trust transfer in the context of AI, thereby extending recent research efforts to understand trust in AI [e.g., 6, $10]$. By converging AI with a base technology while creating an AI-capable technology, multi-source trust transfer offers the theoretical basis to include users' trusting beliefs of multiple sources when examining the trust transfer process. Second, we contribute to trust transfer theory by showing the importance of the duality of trust, whereas the predominance of trust transfer research has either analyzed technology [17] or interpersonal trust transfer [29]. Our study demonstrates that different sources are necessary for multi-source trust transfer depending on the trust perspective. Third, we applied an NCA to help identify which trust sources are necessary conditions for a multi-source trust transfer [20]. In doing so, we not only apply a novel data analysis method but also show its suitability for IS research phenomena. We thereby extend prior trust transfer research taking a subjective logic, relating to mainly argumentative derivation of necessary sources in trust transfer by statistical analysis (e.g., SEM). Indeed, our research highlights that both technology trust sources are needed to achieve users' trust in AV technologies. Studying the effect-sizes and bottleneck table provides detailed insights about the predominance of each trust source, which may also be considered when understanding the transfer of trust in other AI-capable technologies.

For practitioners, our results provide insights into which sources may be necessary to establish trust in AIcapable technologies. AV providers should bear in mind that both technology sources should be considered to understand how users may establish trust in AVs. This suggests that conventional vehicle providers will not only have to address vehicle-specific innovations in terms of trust in technical functionalities but also trust in AI. By contrast, our results may indicate that AI-capable technology providers should ensure that users perceive the relationship with the base technology provider as being necessary compared to the AI provider. Consequently, possible cooperation should continue to center on the base technology provider (i.e., vehicle provider).

\subsection{Limitations and future research}

Our study is subject to limitations that open avenues for future research. First, our study uses the online platform MTurk for the selection of study participants. While prior research acknowledges MTurk's suitability for behavioral studies [e.g., 40], future research should 
employ additional means of data collection. For instance, engaging multiple online panel providers or conducting behavioral experiments could help to triangulate insights. Second, we witnessed minor discriminant validity issues (i.e., between trust in AV technology and AV provider). Future research may compare multi-source trust transfer processes on a provider and technology with general trust perceptions in more detail. Third, NCA is a new technique, and not all issues regarding statistical and causal inference have been resolved. Future research on the statistical properties of estimated ceiling lines and confidence interval estimation is needed further to understand the necessity of conditions [35]. Fourth, we refrained from comparing NCA and SEM results in this study to keep the focus on identifying necessary trust conditions.

With this study, we wanted to create opportunities for behavioral research to yield fresh insight into how to establish trust in AI-capable technologies. In doing so, we also encourage researchers to look at trust transfer theory from a necessity logic to gain deeper insights into how trust is established in related technologies [12]. Future research in multi-source trust transfer may consider a two-step approach (i.e., analyzing the SEM and conducting NCA [28]) to understand which sources are necessary [20]. Finally, future research may take a deeper look at the role of the AI provider in trust transfer to better explain and resolve our surprising findings.

\section{Conclusion}

In this study, we aimed to understand the necessary trust sources to achieve trust in AI-capable technologies. To do so, we contextualized multi-source trust transfer from a dual trust perspective while including AI and vehicle technologies and providers as trust sources and AV technologies and providers as trust target. By conducting an NCA, we revealed the necessity of both trust in vehicle providers and trust in vehicle technologies as a source to transfer trust in AVs. Regarding trust in AI, we show that only trust in AI technology is necessary, whereas trust in AI providers surprisingly is not significant and relevant. We contribute to both research and practice by fostering a deeper understanding of necessary conditions of trust sources in trust transfer. This knowledge can be used to identify how to establish trust in AI-capable technology and ultimately show, with the help of the NCA, how to investigate which sources are necessary for a multi-source trust transfer.

\section{Reference}

[1] Söllner, M., I. Benbasat, J.M. Leinmeister, and P.A. Pavlou, Trust in MIS Quaterly Research Curations, Bush, A. and Rai, A. (eds.), 2016.
[2] Gefen, D., E.W. Karahanna, and D. Straub, "Trust and TAM in Online Shopping: An Integrated Model", MIS Quarterly, 27(1), 2003, pp. 51-90.

[3] Benbasat, I., and W. Wang, "Trust In and Adoption of Online Recommendation Agents", J. Assoc. Inf. Syst., 6(3), 2005, pp. 72-101.

[4] Koester, N., and T.O. Salge, "Building Trust in Intelligent Automation: Insights into Structural Assurance Mechanisms for Autonomous Vehicles", Proc. of ICIS, India, 2020, pp. 2143-2160.

[5] Shin, D., and Y.J. Park, "Role of Fairness, Accountability, and Transparency in Algorithmic Affordance", Comput. Hum. Behav., 98, 2019, pp. 277-284.

[6] Thiebes, S., S. Lins, and A. Sunyaev, "Trustworthy Artificial Intelligence”, Electron. Mark., 31(2), 2020, pp. 447464.

[7] Raisch, S., and S. Krakowski, "Artificial Intelligence and Management: The Automation-Augmentation Paradox", Acad Manage Rev, 46(1), 2021, pp. 192-210.

[8] Pandl, K.D., S. Thiebes, M. Schmidt-Kraepelin, and A. Sunyaev, "On the Convergence of Artificial Intelligence and Distributed Ledger Technology", IEEE Access, 8, 2020, pp. 57075-57095.

[9] Hengstler, M., E. Enkel, and S. Duelli, "Applied Artificial Intelligence and Trust-The Case of Autonomous Vehicles and Medical Assistance Devices", Technol Forecast Soc Change, 105, 2016, pp. 105-120.

[10] Floridi, L., "Establishing the Rules for Building Trustworthy AI", Nat. Mach. Intell., 1(6), 2019, pp. 261-262.

[11] Markus, A.F., J.A. Kors, and P.R. Rijnbeek, "The Role of Explainability in Creating Trustworthy Artificial Intelligence for Health Care", J Biomed Inform, 113, 2021, pp. $1-11$.

[12] Stewart, K.J., "Trust Transfer on the World Wide Web", Organ. Sci., 14(1), 2003, pp. 5-17.

[13] Stewart, K.J., "How Hypertext Links Influence Consumer Perceptions to Build and Degrade Trust Online", J Manag Inf Syst, 23(1), 2006, pp. 183-210.

[14] Glikson, E., and A.W. Woolley, "Human Trust in Artificial Intelligence: Review of Empirical Research", Acad Manag Ann, 14(2), 2020, pp. 627-660.

[15] Lowry, P.B., N.W. Twyman, M. Pickard, J.L. Jenkins, and Q.N. Bui, "Proposing the Affect-Trust Infusion Model (ATIM) to Explain and Predict the Influence of High- and Low-Affect Infusion on Web Vendor Trust", Inf. Manag., 51(5), 2014, pp. 579-594.

[16] Eiband, M., D. Buschek, and H. Hussmann, "How to Support Users in Understanding Intelligent Systems? Structuring the Discussion", Proc. of ACM IUI, College Station, TX, USA 2021, pp. 120-132.

[17] Gong, X., K.Z.K. Zhang, C. Chen, C.M.K. Cheung, and M.K.O. Lee, "What Drives Trust Transfer from Web to Mobile Payment Services?”, Inf. Manag., 57(7), 2020, pp. 1-11.

[18] McKnight, D.H., M. Carter, J.B. Thatcher, and P.F. Clay, "Trust in a Specific Technology: An Investigation of its Components and Measures", ACM Trans Inf Syst, 2(2), 2011, pp. 1-25.

[19] Lansing, J., and A. Sunyaev, "Trust in Cloud Computing: Conceptual Typology and Trust-Building Antecedents", ACM SIGMIS Database, 47(2), 2016, pp. 58-96. 
[20] Dul, J., "Necessary Condition Analysis (NCA): Logic and Methodology of "Necessary but Not Sufficient" Causality”, Organ Res Methods, 19, 2016, pp. 10-52.

[21] SAE, "Taxonomy and Definitions for Terms Related to Driving Automation Systems for On-Road Motor Vehicles", SAE International (J3016), 2018.

[22] Lankton, N.K., D.H. McKnight, and J. Tripp, "Technology, Humanness, and Trust: Rethinking Trust in Technology”, J. Assoc. Inf. Syst., 16(10), 2015, pp. 880-918.

[23] Staples, D.S., and J. Webster, "Exploring the Effects of Trust, Task Interdependence and Virtualness on Knowledge Sharing in Teams”, Inf. Syst. J., 18(6), 2008, pp. 617-640.

[24] Mayer, R.C., J.H. Davis, and F.D. Schoorman, “An Integrative Model of Organizational Trust", Acad Manage Rev, 20(3), 1995, pp. 709-734.

[25] McKnight, D.H., V. Choudhury, and C. Kacmar, "Developing and Validating Trust Measures for e-Commerce", Inf. Syst. Res., 13(3), 2002, pp. 334-359.

[26] Thatcher, J.B., D.H. McKnight, E. White Baker, R. Arsal, and N.H. Roberts, "The Role of Trust in Postadoption IT Exploration”, IEEE Trans Eng Manag, 58(1), 2011, pp. 56-70.

[27] Belanche, D., L.V. Casaló, C. Flavián, and J. Schepers, "Trust Transfer in the Continued Usage of Public e-Services", Inf. Manag., 51(6), 2014, pp. 627-640.

[28] Ringle, C., N. Richter, S. Schubring, S. Hauff, and M. Sarstedt, "When Predictors of Outcomes Are Necessary: Guidelines for the Combined Use of PLS-SEM and NCA", Ind. Manag. Data Syst., 120, 2020, pp. 22432267.

[29] Chen, J., and X.-L. Shen, "Consumers' Decisions in Social Commerce Context: An Empirical Investigation", Decis Support Syst, 79, 2015, pp. 55-64.

[30] Loken, B., L.W. Barsalou, and C. Joiner, Categorization Theory and Research in Consumer Psychology, Lawrence Erlbaum Associates, Mahwah, NJ, 2008.

[31] Cohen, J.B., and K. Basu, "Alternative Models of Categorization: Toward a Contingent Processing Framework”, J. Consum. Res., 13(4), 1987, pp. 455-472.

[32] Lee, K.C., S. Lee, and Y. Hwang, “The Impact of Hyperlink Affordance, Psychological Reactance, and Perceived Business Tie on Trust Transfer", Comput. Hum. Behav., 30, 2014, pp. 110-120.

[33] Pradhan, A., A. Lazar, and L. Findlater, "Use of Intelligent Voice Assistants by Older Adults with Low Technology Use", ACM Trans Comput Hum Interact, 27(4), 2020, pp. 1-27.

[34] Zierau, N., K. Flock, A. Janson, M. Söllner, and J.M. Leimeister, "The Influence of AI-Based Chatbots and Their Design on Users Trust and Information Sharing in Online Loan Applications", Proc. of HICSS, Koloa (Hawaii), USA., 2021, pp. 5483-5492.

[35] van der Valk, W., R. Sumo, J. Dul, and R.G. Schroeder, "When are Contracts and Trust Necessary for Innovation in Buyer-Supplier Relationships?", J. Purch. Supply Manag., 22(4), 2016, pp. 266-277.

[36] Dul, J., E. van der Laan, and R. Kuik, "A Statistical Significance Test for Necessary Condition Analysis", Organ Res Methods, 23(2), 2020, pp. 385-395.

[37] Hair, J., M. Sarstedt, L. Hopkins, and V. Kuppelwieser, "Partial Least Squares Structural Equation Modeling (PLS-SEM): An Emerging Tool for Business Research", Eur. Bus. Rev., 26, 2014, pp. 106-121.

[38] Henseler, J., C.M. Ringle, and M. Sarstedt, “A New Criterion for Assessing Discriminant Validity in Variancebased Structural Equation Modeling", J. Acad. Mark. Sci., 43(1), 2015, pp. 115-135.

[39] McKnight, D.H., P. Liu, and B.T. Pentland, "Trust Change in Information Technology Products", J Manag Inf Syst, 37(4), 2020, pp. 1015-1046.

[40] Lowry, P.B., J. D'Arcy, B. Hammer, and G.D. Moody, "'Cargo Cult' Science in Traditional Organization and Information Systems Survey Research", J. Strateg. Inf. Syst., 25(3), 2016, pp. 232-240.

[41] Peer, E., J. Vosgerau, and A. Acquisti, "Reputation as a Sufficient Condition for Data Quality on Amazon Mechanical Turk", Behav Res Methods, 46(4), 2013, pp. 1023-1031.

[42] Ringle, C., S. Wende, and J.-M. Becker, "SmartPLS 3 (Version 3)", SmartPLS GmbH, Germany, 2015.

[43] Oppenheimer, D.M., T. Meyvis, and N. Davidenko, "Instructional Manipulation Checks", J Exp Soc Psychol, 45(4), 2009, pp. 867-872.

[44] Chin, W.W., J.B. Thatcher, R.T. Wright, and D. Steel, Controlling for Common Method Variance in PLS Analysis: The Measured Latent Marker Variable Approach, Springer, New York, 2013.

[45] Faul, F., E. Erdfelder, A. Buchner, and A.-G. Lang, "Statistical Power Analyses Using G*Power 3.1: Tests for Correlation and Regression Analyses", Behav Res Methods, 41(4), 2009, pp. 1149-1160.

[46] Kline, R.B., Principles and Practice of Structural Equation Modeling (4th ed.), Guilford Press, New York, USA, 2016.

[47] Fornell, C., and D.F. Larcker, "Evaluating Structural Equation Models with Unobservable Variables and Measurement Error", J Mark Res, 18(1), 1981, pp. 39-50.

[48] Nunnally, J.C., Psychometric Theory (2nd ed.), McGrawHill College, New York, NY, 1978.

[49] O’Brien, R.M., “A Caution Regarding Rules of Thumb for Variance Inflation Factors", Quality \& Quantity, 41(5), 2007, pp. 673-690.

[50] Podsakoff, P., S. MacKenzie, J.-Y. Lee, and N. Podsakoff, "Common Method Biases in Behavioral Research", J Appl Psychol, 88(5), 2003, pp. 879-903.

[51] Serrano-Archimi, C., E. Reynaud, H.M. Yasin, and Z.A. Bhatti, "How Perceived Corporate Social Responsibility Affects Employee Cynicism", J. Bus. Ethics, 151(4), 2018, pp. 907-921. 